\title{
Risk factors for gonorrhoea, syphilis, and trichomonas infections among women attending family planning clinics in Nairobi, Kenya
}

\author{
C Costello Daly, N Maggwa, J K Mati, M Solomon, S Mbugua, P M Tukei, D J Hunter
}

Department of Epidemiology, Harvard School of Public Health, Boston, MA, USA

C Costello Daly D J Hunter

Kenya Medical Research Institute, Nairobi, Kenya N Maggwa

J K Mati

P M Tukei

Department of Obstetrics and Gynecology, University of Nairobi, Nairobi, Kenya M Solomon $S$ Mbugua

Channing Laboratory, Brigham and Women's Hospital, Boston, MA, USA 02115

D J Hunter

Correspondence to: Dr D J Hunter, Department of Epidemiology, Harvard School of Public Health, 667 Huntington Avenue Boston, MA 02115, USA.

\begin{abstract}
Objective-To identify the risk factors for gonorrhoea, syphilis, and trichomonas infections among low risk women in Nairobi, Kenya.

Method-In a cross-sectional study, 4,404 women attending two peri-urban family planning clinics between 1989 and 1991 were interviewed using a structured questionnaire and examined for signs of sexually transmitted disease (STD) infection. Cervical cultures for gonorrhoea, PAP smear (including microscopy for trichomonas), RPR and HIV testing were done.

Results-Positive cervical cultures for gonorrhoea were found in $3 \cdot 2 \%$ of women, positive syphilis serology in $1.9 \%$, and positive trichomonas microscopy in $\mathbf{5 \cdot 2 \%}$. Genital ulcers were found in $1.9 \%$ of women. Although unmarried status and reporting more than one sex partner in the previous year were both significantly associated with each disease in the crude analysis, these associations were attenuated after controlling for each other and for other risk factors. The population attributable risks (PARs) for these factors were low (7-16\%) owing to the high proportion of cases who were married and monogamous. The majority of women with microbiological evidence of infection had normal pelvic examinations. Clinical diagnostic algorithms for STDs in this population had a low sensitivity and positive predictive value. Nevertheless, a strong association between HIV seropositivity and STDs was observed.

Conclusion-The low population attributable risks found in this study suggest that behaviour change messages directed to women, particularly if they are married, have a low potential for preventing STDs. The poor performance of clinical diagnostic algorithms illustrates the desirability of testing these algorithms in a variety of populations and reinforces the need for low-cost methods of microbiologic diagnosis if populations with relatively low prevalences of these infections are to be included in programmes to diagnose and treat STDs.
\end{abstract}

(Genitourin Med 1994;70:155-161)
Introduction

The need for the control of sexually transmitted diseases (STDs) has become more urgent since they have been recognised as independent risk factors for HIV acquisition. ${ }^{1}$ Recent prospective studies have demonstrated the role of genital ulcer disease, ${ }^{23}$ gonorrhoea, ${ }^{4}$ chlamydia, ${ }^{34}$ and trichomonas vaginitis ${ }^{4}$ as cofactors in the heterosexual transmission of HIV in Africa. Estimations of the attributable risk of STDs on HIV infections in Africa range from $8 \%$ to $33 \%,{ }^{5}$ indicating that a substantial proportion of HIV infections might be prevented by control of other STDs. ${ }^{6-8}$

In addition, STDs and their complications result in substantial morbidity and mortality independent of their role in HIV transmission. This burden, borne mainly by women and children, includes such life-threatening conditions as ectopic pregnancy, perinatal infections, ophthalmia neonatorum, stillbirth, spontaneous abortion, cervical cancer, low birth weight and congenital infections, in addition to both male and female infertility..$^{9-11}$

In Africa, a high prevalence of STDs is often observed even among women usually considered to be at a low risk for STDs. Village surveys and studies among antenatal and family planning clinic attenders and postpartum women report infection rates ranging from $2 \%-22 \%$ for gonorrhoea, $0 \cdot 3 \%-22 \%$ for syphilis, $6 \%-29 \%$ for chlamydia, and $5 \%-49 \%$ for trichomonas vaginitis..$^{9-11}$ Current facilities for STD diagnosis and treatment in developing countries are inadequate and it has been recommended that STD care be integrated into maternal and child health care and family planning services. ${ }^{8}$

Numerous prevalence studies have been done in Africa, but fewer attempts have been made to identify the behavioural and biological risk factors for sexually transmitted diseases other than HIV in "low-risk" women. ${ }^{12-18}$ To further investigate risk factors for STDs among women of reproductive age not considered to be members of high risk groups, we conducted interviews and examinations at two family planning clinics in Nairobi, Kenya.

\section{Methods}

Study population As previously described, we enrolled women attending two peri-urban family planning clinics serving mainly lower 
Table 1 Relationships between socio-demographic, sexual behaviour, and physical examination variables and positive cervical cultures for Neisseria gonorrheae among 4165 women attending two family planning clinics in Nairobi, Kenya

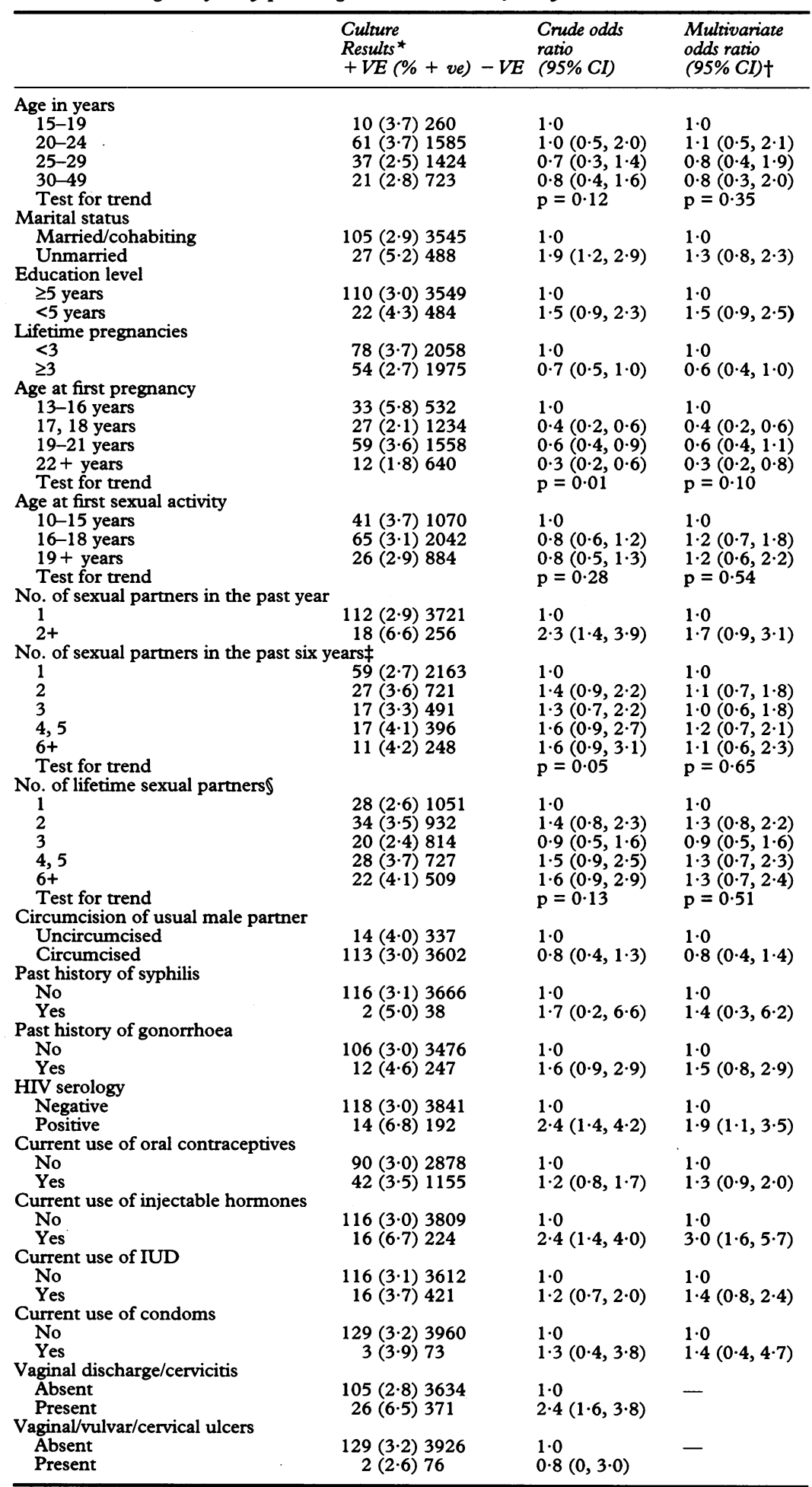

${ }^{\star}$ Numbers do not always add to 132 gonorrhoea culture positives and 4033 culture negatives due to missing values.

†From a multivariate logistic regression model including indicator variables for: age, marital status, education level, number of lifetime pregnancies, age at first pregnancy, age at first intercourse, number of sex partners in the past year, circumcision status of usual male partner, history of syphilis infection, history of gonorrhoea infection, HIV serologic status, current use of ora contraceptives, current use of injectable hormones, current use of IUD, current use of condoms. $\ddagger$ From the above multivariate logistic regression model with number of sexual partners in the past six years replacing number of sexual partners in the past year.

§From the above multivariate logistic regression model with number of lifetime sexual partners replacing number of sexual partners in the past year.

socio-economic status areas of Nairobi between October 1989 and May $1991 .{ }^{19}$ Signed informed consent for HIV and STD screening was obtained after HIV pre-test counselling. Over $90 \%$ of women approached agreed to participate and there were no signif- icant differences between women who agreed and those who refused with relation to age, marital status, and parity. ${ }^{20}$

Information on social, demographic, medical, and sexual behaviour characteristics was obtained by a pre-tested structured questionnaire administered by trained interviewers. Each participant underwent a general medical examination and pelvic examination, at which time endocervical swabs for gonorrhoea cultures and scrapings (using an Ayre's wooden spatula) for PAP smears were taken. Blood was drawn for HIV testing and syphilis serology. A follow-up appointment was made for post-test counselling and treatment of and referral for any identified STDs or abnormal PAP smears.

Laboratory methods Specimens for gonorrhoea were inoculated directly onto ThayerMartin culture medium plates at the clinics. The plates were transported to the laboratory on the same day and incubated at $36-37^{\circ} \mathrm{C}$. The oxidase test and Gram stain were used to identify positive Neisseria gonorrhoeae cultures. Syphilis serology was assayed using the rapid plasma reagin (RPR) test (Becton Dickinson Microbiology Systems). Infection with Trichomonas vaginalis was identified by the presence of trichomonads on the PAP smear. HIV serology was initially tested by ELISA (using commercially available kits) and positive sera were confirmed by Western Blot (Dupont de Nemours, Wilmington, DE). All laboratory tests were performed by technicians who were unaware of the questionnaire or examination findings. Gonorrhoea results (239), 37 syphilis results, and 496 trichomonas results were unavailable owing to laboratory error or unsatisfactory PAP smears.

Statistical methods Odds ratios (hereafter referred to as relative risks ${ }^{21}$ ) and their $95 \%$ confidence intervals were calculated using STATA Statistical Software ${ }^{22}$; exact confidence intervals were calculated when appropriate using Epi Info. ${ }^{23}$ The Mantel test for trend in ordered categorical variables in the univariate analysis was also calculated using Epi Info. ${ }^{24}$ Multivariate logistic regression was used to control for confounding factors using STATA. The choice of variables in the model was based on both the results of the univariate analyses and past studies of risk factors for STDs. To maximise comparability of risk factors for the three STDs, we present multivariate analyses for each STD controlling for the same set of observed and potential risk factors. Calculation of population attributable risks (PARs) was based on odds ratios generated by the crude analysis. ${ }^{5}$

\section{Results}

Among the 4,404 women enrolled in the study, the prevalence of sexually transmitted diseases was as follows: positive cervical gonorrhoea cultures, $3 \cdot 2 \%$ (132 out of 4165 with an available result, $95 \% \mathrm{CI} 2 \cdot 6-3 \cdot 7)$; syphilis seropositivity, $1.9 \%$ ( 83 out of 4367 with an available result, $95 \%$ CI $1 \cdot 5-2 \cdot 4)$; and posi- 
Table 2 Relationship between socio-demographic, sexual behaviour, and physical examination variables and positive cervical cultures for positive syphilis serology among 4367 women attending two family planning clinics in Nairobi, Kenya

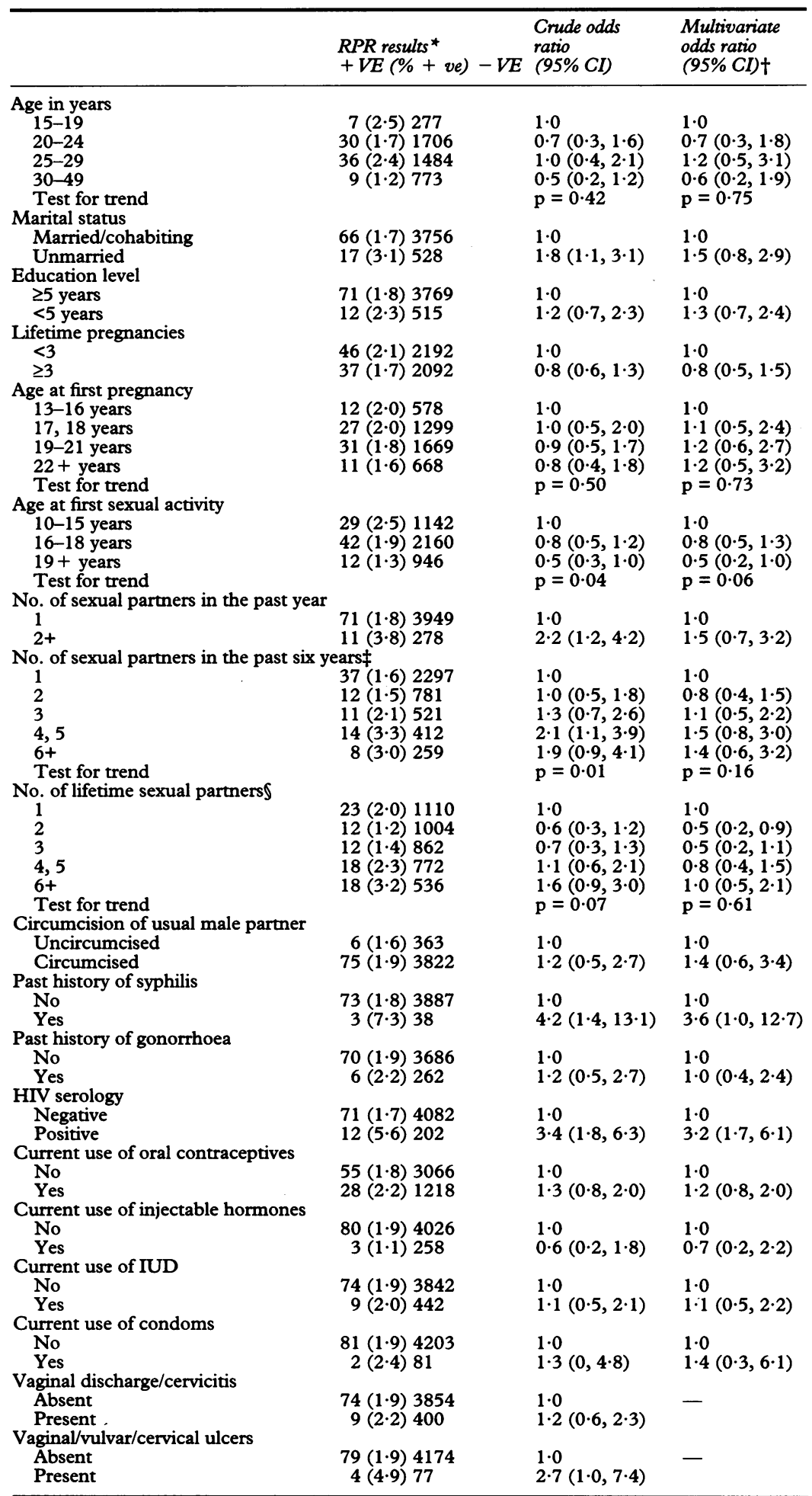

* Numbers do not always add to 83 RPR positives and 4284 RPR negatives due to missing values tFrom a multivariate logistic regression model including indicator variables for: age, marital †From a multivariate logistic regression model including indicator variables for: age, marital
status, education level, number of lifetime pregnancies, age at first pregnancy, age at first interstatus, education level, number of lifetime pregnancies, age at first pregnancy, age at first inter-
course, number of sex partners in the past year, circumcision status of usual male partner, history course, number of sex partners in the past year, circumcision status of usual male partner, history
of syphilis infection, history of gonorrhoea infection, HIV serologic status, current use of oral contraceptives, current use of injectable hormones, current use of IUD, current use of condoms. †From the above multivariate logistic regression model with number of sexual partners in the past six years replacing number of sexual partners in the past year.

§From the above multivariate logistic regression model with number of lifetime sexual partners replacing number of sexual partners in the past year.

tive trichomonas microscopy, $5 \cdot 2 \%$ (205 out of 3908 with an available result, $95 \%$ CI $4 \cdot 6-6 \cdot 0) .4 \cdot 9 \%(216$ out of $4404,95 \%$ CI $4 \cdot 3-5 \cdot 5)$ of women were HIV seropositive.

Syphilis seropositivity was positively associated with both gonorrhoea infection ( RR 2.5, $95 \% \mathrm{CI} 1 \cdot 1-5 \cdot 8)$ and trichomonas vaginitis
(RR 1·6, 95\% CI 0·7-3·6). Gonorrhoea and trichomonas infections were not positively associated ( RR 0.8, 95\% CI 0.3-2.0). Few women were found to have evidence of more than one disease at the time of the study. Of women who were gonorrhoea positive, only $2 \%$ (3/132) also had a positive RPR. Of women with positive trichomonas microscopy, 3\% (6/205) had a positive RPR, and $2 \%(5 / 205)$ had positive gonorrhoea cultures. Only one study participant had all three outcomes simultaneously.

None of the STDs studied were significantly related to age or educational level in this population. Eighty eight percent of the women were married. Owing to the low number of unmarried women, we were not able to examine the component categories single, widowed, and divorced separately. Ninety three percent of all women and $97 \%$ of married women reported only a single sexual partner in the past year. Only $2 \cdot 7 \%$ reported having accepted money for sex at any time. Having more than one sex partner in the past year and being unmarried were both significantly associated with each STD in the crude analyses (Tables 1, 2, 3). However, these associations were not significant in the multivariate analysis (except for the association between being unmarried and trichomonas), largely because of mutual confounding of number of sex partners and marital status. The crude PAR for unmarried women vs. married women was $9.7 \%$ for gonorrhoea, $\mathbf{9} \cdot 1 \%$ for syphilis, and $\mathbf{1 5} \cdot \mathbf{9} \%$ for trichomonas.

The crude PARs for more than one sex partner in the past year are of similarly low magnitude: $7 \cdot 7 \%$ for gonorrhoea, $7 \cdot 2 \%$ for syphilis, and $7 \cdot 4 \%$ for trichomonas. The number of sex partners in the past year was more strongly associated with current STDs than the number of sex partners in the past six years or number of lifetime sex partners.

Later age at first sexual intercourse was weakly inversely associated with gonorrhoea and trichomonas infections and significantly inversely associated with syphilis seropositivity $(p=0.04$ in the crude analysis, $p=0.06$ in the multivariate analysis). Older age at first pregnancy was inversely associated with a positive gonorrhoea culture. The test for trend was not significant in multivariate analysis, but the relative risks for 17-18 years of age and for $22+$ years of age (both compared to 13-16 years) remained significantly inverse. Age at first pregnancy was not significantly associated with either syphilis or trichomonas.

A past history of gonorrhoea infection was reported by $6 \%$ of women and a past history of syphilis was reported by $1 \%$. In the multivariate analysis, a history of gonorrhoea was positively associated with current evidence of gonorrhoea infection, but not with positive syphilis serology or trichomonas microscopy. Similarly, a reported past history of syphilis was significantly associated with positive syphilis serology. Only $8 \% \quad(11 / 132)$ of women with positive gonorrhoea cultures reported a history of gonorrhoea in their usual male partner, $12 \%$ reported no past history in 
Table 3 Relationship between socio-demographic, sexual behaviour, and physical examination variables and positive cervical cultures for positive trichomonas microscopy among 3908 women attending two family planning clinics in Nairobi, Kenya

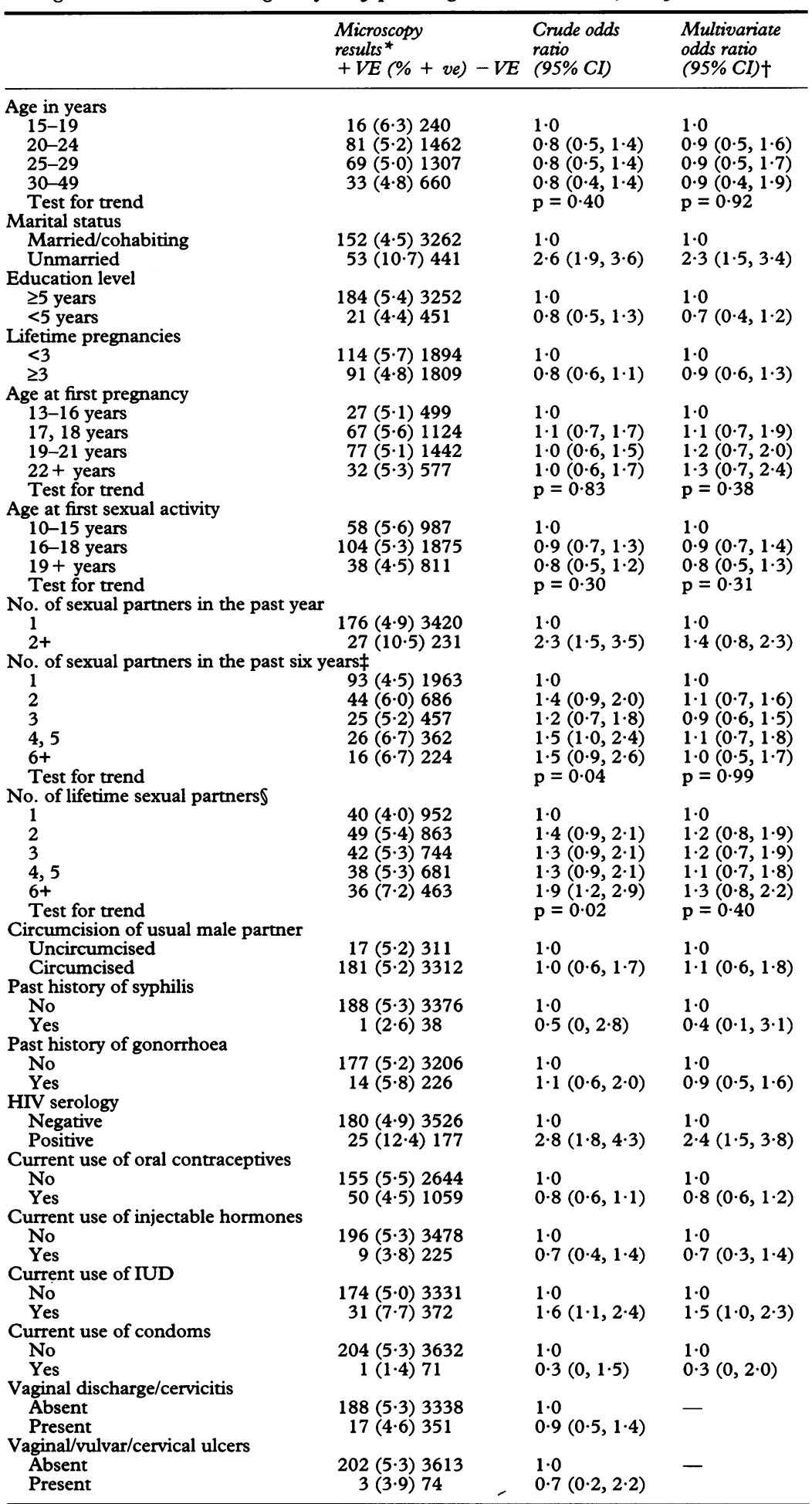

^Numbers do not always add to 205 microscopy positives and 3703 microscopy negatives due to missing values.

†From a multivariate logistic regression model including indicator variables for: age, marita status, education level, number of lifetime pregnancies, age at first pregnancy, age at first intercourse, number of sex partners in the past year, circumcision status of usual male partner, histor of syphilis infection, history of gonorrhoea infection, HIV serologic status, current use of ora contraceptives, current use of injectable hormones, current use of IUD, current use of condoms. $\ddagger$ From the above multivariate logistic regression model with number of sexual partners in th past six years replacing number of sexual partners in the past year.

SFrom the above multivariate logistic regression model with number of lifetime sexual partners replacing number of sexual partners in the past year.

their partner, and $80 \%$ felt unable to assess their partner's history. These percentages were not materially different in analyses restricted to married women only.

No strong associations were observed between the type of contraceptive method used and the three STDs studied, with the exception of a significant positive association between the current use of injectable hormones and gonorrhoea infection (multivariate $\mathrm{RR}=3 \cdot 0,95 \% \mathrm{CI} 1 \cdot 6-5 \cdot 7)$ and the current use of an IUD and trichomonas infection (multivariate $\mathrm{RR}=1 \cdot 5,95 \%$ CI $1 \cdot 0-2 \cdot 3$ ). Duration of past injectable hormone use was positively but not significantly related to the gonorrhoea risk (crude RRs 1.9 for 1-12 months and 1.5 for $13-65$ months, $p$ value for trend $=0.07$ ). Similarly, the duration of past IUD use was not significantly related to the risk of trichomonas infection (crude RRs $1 \cdot 1$ for 1-12 months and 1.6 for 13-66 months, $p$ value for trend $=0 \cdot 14$ ).

The most significant predictor of gonorrhoea, syphilis, and trichomonas infections in both the crude and multivariate analyses was HIV seropositivity. This association is reported in detail elsewhere.$^{25}$ Interestingly, a strong positive association between a woman's usual male partner being circumcised and HIV seropositivity was observed, but we did not observe any strong associations between partner's circumcision and these three STDs in the same population.

Genital ulcers were present in $1.9 \%$ of women in the study and were positively associated with syphilis seropositivity. Clinical evidence of vaginal or cervical inflammation (vaginal discharge or cervicitis) on examination was present in $9.4 \%$ of study participants and was significantly positively associated with positive gonorrhoea cultures (RR 2.4, $95 \%$ CI $1 \cdot 6-3 \cdot 8$ ), but not syphilis or trichomonas.

The sensitivity, specificity, and positive predictive value of being unmarried, having more than one sex partner in the past year, and having signs on physical examination (either genital ulcer or inflammation) for each of the STDs are presented in table 4. Risk factors are considered singly and then combined into risk profiles. In general, the sensitivity and positive predictive value of individual and combined variables was low and the specificity was high.

\section{Discussion}

Even in this relatively low-risk population attending family planning clinics, we observed a substantial burden of sexually transmitted diseases. However, few historical, demographic, or clinical factors were predictive of STDs. Due to the low prevalence of high-risk behaviours and positive examination findings, only a minority of women with STDs would be identified if screened according to the presence of such factors.

The prevalences of STDs and HIV observed in this study are.similar, although slightly lower than those seen in studies of STDs in obstetric populations in Nairobi. ${ }^{16} 17$ 26-28 Not all of those studies measured risk factors, but those that did included a higher proportion of women with risk factors than we observed, such as being unmarried, reporting a past STD infection, ${ }^{1626}$ or reporting more 
Table 4 Sensitivity, specificity, and positive predictive value of selected history and physical examination variables associated with sexually transmitted diseases

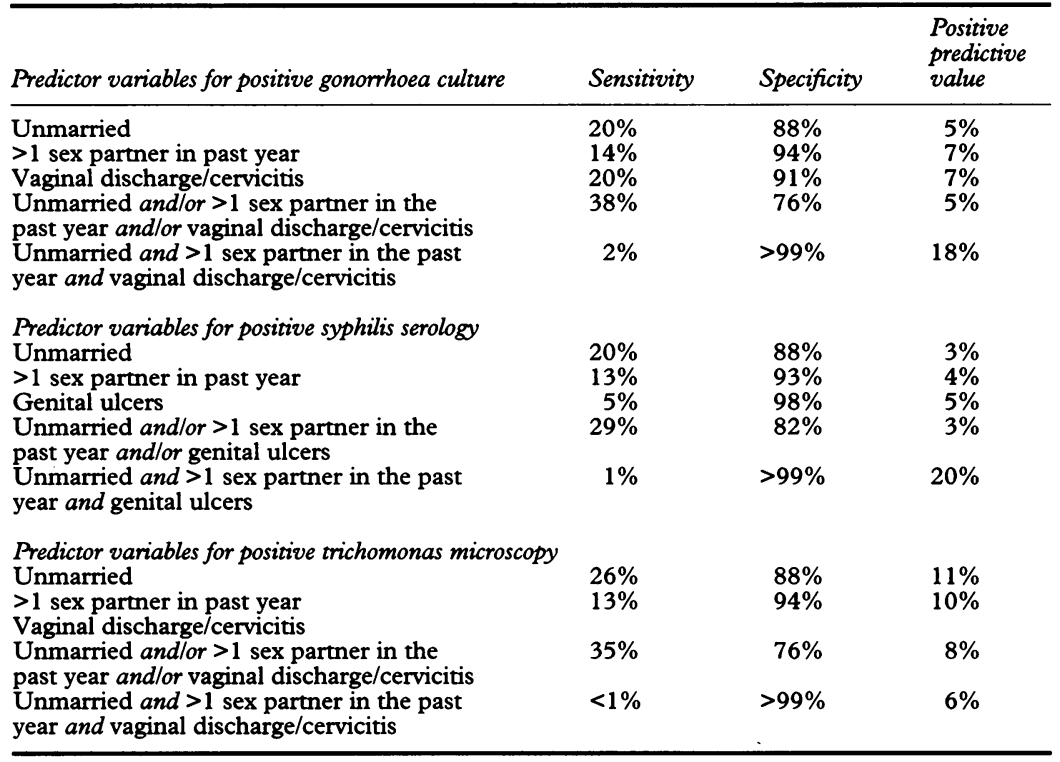

than one recent sex partner. ${ }^{26}$ In addition, area of residence within Nairobi has previously been demonstrated to be an independent predictor of upper genital tract infection. ${ }^{17}$ The slightly lower prevalences of STDs in our study may be due to geographic variation in STD risk factor prevalence, or the fact that family planning clinics and obstetric clinics sample different source populations.

Both having more than one sex partner in the past year and being unmarried were significant predictors of all three STD outcomes, although these were not independent in the multivariate analysis. Since the majority of women with STDs were married and monogamous, the crude population attributable risks for unmarried status and more than one sex partner in the past year ranged from $7 \%$ to $16 \%$. Only $7 \%$ of all women in the study reported more than one sex partner in the past year, consistent with other studies of the selfreported sexual behaviour of women in the general population in Africa. ${ }^{29}$

The scarcity of facilities and personnel available for the microbiologic diagnosis of STDs in developing countries means that although screening women in the general population for STDs is highly desirable, it is not currently feasible or affordable. Although the World Health Organization has recommended diagnostic algorithms based on clinical symptoms for the treatment of STDs, ${ }^{30}$ casefinding for asymptomatic STDs presents a more difficult challenge. Few tests of this approach have been conducted in general populations. Braddick et $a l^{16}$ developed a diagnostic algorithm to identify pregnant women at risk of cervical infection in a Nairobi hospital and were able to predict a combined endpoint of either gonorrhoea and/or chlamydial infection (combined prevalence $16 \%$ ) using history and speculum examination findings with a sensitivity of $68 \%$, a positive predictive value of $35 \%$, and specificity of $93 \%$. Vuylsteke et $a l^{18}$ developed a scoring system based on five epidemiologic and historical risk factors and a urine leukocyte-esterase dipstick (for polymorphonuclear leukocytes) which gave a sensitivity of $72 \%$, a positive predictive value of $16 \%$, and a specificity of $74 \%$ in the diagnosis of gonorrhoea and/or chlamydial infection in pregnant women (combined prevalence $6.5 \%$ ) in Kinshasa.

In our study, the construction of diagnostic algorithms using the most readily accessible and significant risk factors and physical examination findings gave lower sensitivities and positive predictive values, although specificities remained high. The highest sensitivity of $38 \%$ was found for any combination of unmarried status, more than one sex partner in the previous year, and evidence of vaginal or cervical infection on physical examination in the diagnosis of cervical gonorrhoea infection, but the positive predictive value was low (5\%). The prevalence of historical risk factors was lower in our study than in other studies; this would be expected to reduce the observed sensitivity and increase the specificity of these factors in the diagnosis of each STD. Furthermore, we used single STDs as the outcome, in contrast to a combination of gonorrhoea and/or chlamydia as in the previous studies, which would be expected to lower the performance of the algorithms in this study. Some of the women who fit the risk profiles but did not have one of the three STDs in this study may have had cervical chlamydial infection: if we had also screened for chlamydia, we would expect that the positive predictive value of each algorithm would be higher for a combined outcome. The effect of the addition of chlamydia diagnosis on sensitivity and specificity however, would depend on the performance of the particular algorithm for chlamydia.

Of the women with positive gonorrhoea cultures $62 \%$ ( 82 out of 132) did not fit any of the risk profiles, even those based on a single factor. This suggests that the identification of women for diagnostic testing or empirical treatment based on risk factors, although perhaps the best option in some settings, especially those in which the prevalence of risk factors and/or STDs is high, will miss most cases of disease in lower risk populations. Further studies to optimise risk profiles may increase their clinical usefulness in casefinding.

Despite the high level of awareness and knowledge of gonorrhoea in this population, ${ }^{31}$ only $8 \%$ of women with positive gonorrhoea cultures reported a history of gonorrhoea in their partner. A history of gonorrhoea in their partner was denied by $12 \%$ of infected women and $80 \%$ did not feel able to assess their partner's history. This highlights the difficulty of identifying women at risk through their knowledge of their male partner's history. Since men are more often symptomatic with STDs and are more likely to present to an STD clinic, referral of their asymptomatic female partners may provide an additional approach to identifying disease in this population of women. Further studies to identify 
effective and culturally acceptable methods of partner referral in developing countries are needed.

Conducting this study in a family planning clinic offered an unusual opportunity to investigate the associations between contraceptive methods and STDs. In general, there was little association found. However, we observed a positive association between IUD use and trichomonas infection. A single published study $^{32}$ reported a similar (but non-significant) association, suggesting that this deserves further study. In addition, a positive association between injectable contraceptive use and gonorrhoea was found, but this was not clearly related to the duration of use. This association has not been found in previous studies and it is not clear what biological mechanism could account for it. This association may be due to chance or to unknown confounding factor(s) in this population. It is also worth noting the very low rate of condom use in this population, even among women who do not desire pregnancy at the time of the study.

The associations found here between risk factors and these STDs are weaker than those observed between these risk factors and HIV seropositivity in this population. ${ }^{25}$ Since selfreported behaviour information in this population is similar to that seen in other studies, ${ }^{29}$ this is unlikely to be due to the effect of the interviewing methodology. It may be because of a different epidemiology of non-HIV STDs, but is likely to be at least partly due to the fact that these STDs are curable, whereas HIV is not. Treatment, by converting women from positive to negative for current STDs would attenuate associations with risk factors which would be seen if longer-term measures of STD occurrence were available. We did not have information about past STD treatment; however, the effect of recent treatment may not be substantial since STD screening and treatment are not routine in Nairobi at either family planning or antenatal clinics and relatively few other options for treatment exist. Also, women reported a low frequency of past history of STDs, suggesting that most had not received a recent specific diagnosis of STD. Informal treatment, or antibiotic use for other conditions, might still be responsible for lowering the prevalence of these STDs at the time of examination.

In addition, non-differential misclassification of the laboratory diagnosis of these STDs may have biased these associations towards the null, especially in the case of trichomonas and syphilis. The diagnosis of Trichomonas vaginalis either by wet mount or by Papanicolaou smears has a low sensitivity (50-60\%) compared with culture, and cytologic smear diagnosis has a slightly lower. specificity compared with wet mount ( $99 \%$ vs. $100 \%) .{ }^{33} 34$ Syphilis diagnosis in this study relied on the RPR card test with no confirmational treponemal tests. Although there is almost certainly some misclassification due to false positive RPRs, syphilis seropositivity can be thought of as a more long-term marker in this study than gonorrhoea or trichomonas diagnosis. In addition, since the duration of a reactive RPR card test varies with many factors including treatment, a positive $R P R$ could indicate either present or past infection. This may account for the stronger association for syphilis seen for past behaviour variables, such as age at first sexual intercourse, compared to the associations with gonorrhoea or trichomonas. Finally, the relative risks found in this study between STDs and HIV ${ }^{25}$ are similar to those found in other studies in Africa, ${ }^{41526273536}$ suggesting that our laboratory diagnosis of these STDs was reasonably accurate.

In summary, as the prevalence of STDs and HIV in women in the general population continues to rise, the importance of diagnosing and treating STDs increases. Since many STDs in women are asymptomatic, active case-finding is necessary. Unfortunately, although several risk factors studied here were associated with STDs, none were sufficiently predictive, even in combination, to be useful as screening tools for the diagnosis of STDs in this population. The low PARs found in this study suggest that the potential preventive effect of behavioural change messages directed at women is low, particularly if they are married, and such behaviour change messages could be more productive if directed toward their male partners. In the meantime, inexpensive diagnostic tests are urgently needed to take advantage of the opportunity to treat women with STDs in family planning and other clinics.

We are grateful To Dr Pius Achola, formerly of the Nairobi City Commission, for permission to work in the City Commission clinics, as well as the efforts of the nurses and medical personnel involved in data collection.

This study received financial support from the Rockefeller This study received financial support from the Rockefeller
and Ford Foundations through the AIDS and Reproductive and Ford Foundations through the AIDS and Reproductive
Health Network. N. Maggwa was partially supported by a Health Network. N. Maggwa was partially supported by a
training grant from the Fogarty International Center, National training grant from the Fogarty International Center, National
Institutes of Health (D43 TW 00004) to the Harvard AIDS Institutes

1 Laga M. Human immunodeficiency virus infection prevention: the need for complementary sexually transmitted disease control. In: Germain A, Holmes KK, Piot P, Wasserheit JN, Eds. Reproductive Tract Infections: Global Impact and Priorities for Women's Reproductive Health. New York, Plenum Press 1992.

2 Cameron DW, Simonsen IN, D'Costa LJ, et al. Female to male transmission of human immunodeficiency virus type 1: risk factors for seroconversion in men. Lancet type 1: risk fact

3 Plummer FA, Simonsen IN, Cameron DW, et al. Cofactors in male to female transmission of human immunofeficiency virus type 1. F Infect Dis 1991;163:233-9.

4 Laga M, Manoka A, Kivuvu M, et al. Non-ulcerative sexually transmitted diseases as risk factors for HIV-1 transmission in women: results from a cohort study. AIDS 1993;7:95-102.

5 Berkley SF. The public health significance of sexually transmitted diseases in HIV infection in Africa. In: Chen LC, Sepulveda Amor J, Segal SJ, eds. AIDS and Women's Reproductive Health. New York, Plenum Press 1991.

6 Pepin J, Plummer FA, Brunham RC, Piot P, Cameron DW, Ronald AR. The interaction of HIV infection and other sexually transmitted diseases: an opportunity for intervention. AIDS 1989;3:3-9.

7 Potts M, Anderson R, Boily M-C. Slowing the spread of human immunodeficiency virus in developing countries. human immunodeficiency

8 Merson MH. Slowing the spread of HIV: agenda for the 1990s. Science 1993;260:1266-8.

9 Wasserheit JN, Holmes KK. Reproductive tract infections: challenges for international health policy, programs, and research. In: Germain A, Holmes KK, Piot P, Wasserheit JN, eds. Reproductive Tract Infections: Global Impact and Priorities for Women's Reproductive Health. New York, Plenum Press 1992. 
10 Schulz KF, Cates W, O'Mara PR. Pregnancy loss, infant death, and suffering: legacy of syphilis and gonorrhoea in Africa. Genitourin Med 1987;63:320-5.

11 Brunham RC, Embree JE. Sexually transmitted diseases: current and future dimensions of the problem in the third world. In: Germain A, Holmes KK, Piot P, Wasserheit NN, eds. Reproductive Tract Infections: Wasserheit JN, eds. Reproductive Tract Infections: Global Impact and Priorities for Women's

12 Duncan ME, Tibaux G, Pelzer A, et al. First coitus before menarche and risk of sexually transmitted disease. Lancet 1990;335:338-40.

13 Duncan ME, Reimann K, Tibaux G, Pelzer A, Mehari L, Lind I. Seroepidemiological study of gonorrhoea in Ethiopian women. 2. Socioeconomic picture. Genitourin Med 1991;67:493-7.

14 Duncan ME, Jamil Y, Tibaux G, Pelzer A, Mehari L, Darougar S. Seroepidemiological and socioeconomic studies of genital chlamydial infection in Ethiopian women. Genitourin Med 1992;68:221-7.

15 Zewdie D, Tafari N. Human immunodeficiency virus and syphilis infection in women of childbearing age in Addis Ababa, Ethiopia. In: Chen LC, Sepulveda Amor J, Segal SJ, eds. AIDS and Women's Reproductive Health. New York, Plenum Press 1991 .

16 Braddick MR Ndinya-Achola JO, Mirza NB, et al. Towards developing a diagnostic algorithm for Chlamydia trachomatis and Neisseria gonorrhoeae cervicitis in pregnancy. Genitourin Med 1990;66:62-5.

17 Plummer FA, Laga M, Brunham RC, et al. Postpartum upper genital tract infections in Nairobi, Kenya: epidemiology, etiology, and risk factors. $\mathcal{F}$ Infect Dis 1987 ; 156:92-8.

18 Vuylsteke B, Laga M, Alary M, et al. Clinical algorithms for the screening of women for gonococcal and chlamydial infection: evaluation of pregnant women and prosti-

19 Maggwa N, Hunter DJ, Mbugua S, Tukei P, Mati JK. The relationship between human immunodeficiency virus (HIV) infection and cervical intraepithelial neoplasia (CIN) among women attending two family planning (CIN) among women attending two family

20 Maggwa N, Hunter DJ, Mati JK, et al. Acceptability of screening for HIV infection among women attending two family planning clinics in Nairobi, Kenya. Abstract SC 669 , VI International Conference on AIDS, San Francisco, CA, June 1990

21 Rothman KJ. Modern Epidemiology. 1st ed. Boston MA Little, Brown, 1986.

22 Computing Resource Center. Stata Reference Manual: Release 3. 5th ed Santa Monica, CA 1992.
23 Dean AG, Dean JA, Burton AH, Dicker RC. Epi Info, Version 5: a word processing, database, and statistics program for epidemiology on microcomputers. Stone Mountain, GA, USD, Inc. 1990.

24 Schlesselman JJ. Case-control Studies. New York, Oxford University Press 1982.

25 Hunter DJ, Maggwa N, Mati JKG, Tukei PM, Mbugua S. Sexual behavior, STDs, male circumcision, and risk of Sexual behavior, STDs, male circumcision, and risk of 1994;8:93-9.

26 Temmerman $M$, Mohamed Ali F, Ndinya-Achola J, Moses S, Plummer FA, Piot P. Rapid increase of both HIV-1 infection and syphilis among pregnant women in Nairobi, Kenya. AIDS 1992;6:1181-5.

27 Braddick MR, Kreiss JK, Embree JE, et al. Impact of maternal HIV infection on obstetrical and early neonatal outcome. AIDS 1990;4:1001-5.

28 Laga M, Plummer FA, Nzanze H, et al. Epidemiology of ophthalmia neonatorum in Kenya. Lancet 1986;ii:1145-9.

29 Hunter DJ. AIDS in Sub-Saharan Africa: The epidemiology of heterosexual transmission and the prospects for prevention. Epidemiology 1993;4:63-72.

30 World Health Organization. Management of patients with sexually transmitted diseases. Report of a WHO Study sexually transmitted diseases. Report of a WHO Study

31 Garland M, Maggwa BN, Mati JK, et al. Knowledge of AIDS and other sexually transmitted diseases among women attending a family planning clinic in Nairobi, Kenya. Am ₹ Prev Med 1993;9:1-5

32 Avonts D, Sercu M, Heyerick P, Vandermeeren I, Meheus A, Piot $P$. Incidence of uncomplicated genital infections in women using oral contraception or an intrauterine device: a prospective study. Sexually Transmitted Diseases 1990;17:23-9.

33 Krieger JN, Tam MR, Stevens CE, et al. Diagnosis of trichomoniasis. Comparison of conventional wet-mount examination with cytologic studies, cultures, and monoclonal antibody staining of direct specimens. $\mathfrak{F} A M A$ 1988;259:1223-30.

34 Rein MF, Muller M Trichomonas vaginalis and trichomoniasis. In: Holmes KK, Mardh PA, Sparling PF niasis. In: Holmes KK, Mardh PA, Sparling PF, Wiesner PJ, eds. Sexually Transmitted

35 Kreiss JK, Koech D, Plummer FA, et al. AIDS virus infection in Nairobi prostitutes. Spread of the epidemic to East Africa. N Engl Y Med 1986;314:414-8.

36 Kapiga SH, Shao JF, Lwihula GK, Hunter DJ. Risk factors for HIV infection diseases (STD's) among women attending family planning clinics in Dar-esSalaam, Tanzania. $\mathcal{f}$ Acq Immune Deficiency Syndr (in press). 